\title{
Las tecnologías de la información y la comunicación y su relación con la gestión del talento humano
}

\begin{abstract}
Information and Communication Technologies and their relationship with the management of Human Talent
\end{abstract}

Marco Antonio Gavilanes Sagñay. ${ }^{1}$, Fredy Gavilanes-Sagnay. ${ }^{2,3} \&$ Nathalie Azucena Chávez Granados. ${ }^{4}$

\begin{abstract}
DOI: https://doi.org/10.33262/concienciadigital.v4i4.2.1961

Introduction. Information and Communication Technologies have impacted all areas of human and social development. In the case of Human Talent Management, it continues to be a topic of interest, however, it is not always possible to visualize what to do in order to optimize the management of human talent from the perspective of Information and Communication Technology. Target. Establish the relationship between Information and Communication Technologies and the quality of the Human Talent Management process. Methodology. The study used a descriptive, nonexperimental methodology based on theoretical methods that allowed us to review the preceding literature and establish the main arguments related to the topic. Results. 1. The theoretical foundations confirm the importance of using Information and Communication Technologies as a fundamental tool to improve Human Talent Management in different institutions. 2. Work is required to eliminate digital gaps,

\footnotetext{
${ }^{1}$ Escuela Superior Politécnica de Chimborazo (ESPOCH), Facultad de Administración de Empresas, Riobamba, Chimborazo, Ecuador, marco.gavilanes@espoch.edu.ec, https://orcid.org/0000-0002-74703732

${ }^{2}$ Departamento de Ciencias de la Computación, Universidad de las Fuerzas Armadas ESPE sede Santo Domingo, Santo Domingo, Ecuador, fmgavilanes1 @espe.edu.ec, https://orcid.org/0000-0001-5494-374X

${ }^{3}$ Departamento de Informática y Ciencias de la Computación, Facultad de Ingeniería de Sistemas, Escuela Politécnica Nacional, Quito, Ecuador, fredy.gavilanes@epn.edu.ec, https://orcid.org/0000-00015494-374X

4 Investigador Independiente, Riobamba, Chimborazo, Ecuador, nathaliechavezg@gmail.com, https://orcid.org/0000-0002-4764-9160
} 
which still persist; This implies achieving accessibility to computers, the Internet and the technology itself, eliminating the existing inequality. 3. An integrated development strategy is proposed in five phases: Diagnosis, Planning, Application, Reorganization, which allows optimizing the Human Talent Management process from the perspective of Information and Communication Technology. Conclusions. A review of the theoretical references confirms the importance of using Information and Communication Technology as a fundamental tool for improving Human Talent Management in different institutions or companies. A possible development strategy is provided to be applied in the different ones for the optimization of the development of competences and management of ICTs, with a view to improving Human Talent Management.

Keywords: Information and Communication Technologies, Management and Human Talent.

\section{Resumen}

Introducción. Las Tecnologías de la Información y la Comunicación han impactado en todas las áreas del desarrollo humano y social. En el caso de la Gestión del Talento Humano, continúa siendo un tema de interés, sin embargo, no siempre se alcanza a visualizar qué hacer para poder optimizar la gestión del talento humano desde la perspectiva de la Tecnología de la Información y la Comunicación. Objetivo. Establecer la relación que se da entre las Tecnologías de la Información y la Comunicación y la calidad del proceso de Gestión del Talento Humano. Metodología. En el estudio se utilizó una metodología descriptiva, no experimental basada en métodos teóricos que permitieron revisar la literatura precedente y establecer los principales argumentos relacionados con el tema. Resultados. 1. Los fundamentos teóricos confirman la importancia de utilizar las Tecnologías de la Información y la Comunicación como herramienta fundamental para perfeccionar la Gestión del Talento Humano en las diferentes instituciones. 2. Se requiere trabajar en la eliminación de las brechas digitales, que aún persisten; ello implica lograr la accesibilidad a computadoras, internet y a la tecnología en sí, eliminando la desigualdad existente. 3. Se propone una estrategia de desarrollo integrada en cinco fases: Diagnóstico, Planificación, Aplicación, Reorganización, que permite optimizar el proceso la Gestión del Talento Humano desde la perspectiva de la Tecnología de la Información y la Comunicación. Conclusiones. Se revisión de los referentes teóricos confirman la importancia de utilizar la Tecnología de la Información y la Comunicación como herramienta fundamental para la mejora de la Gestión del Talento Humano en las diferentes instituciones u empresas. Se aporta una estrategia de desarrollo posible a aplicar en las diferentes para la optimización del desarrollo de las competencias y manejo de la las TICs, con vista al perfeccionamiento de la Gestión del Talento Humano.

Palabras clave: Tecnologías de la Información y la Comunicación, Gestión y Talento Humano. 


\section{Introducción}

La llegada de las Tecnologías de la Información y la Comunicación ha impactado en todas las áreas del desarrollo humano y social. Desde la mitad del siglo pasado las llamadas Nuevas Tecnologías de la Información y la Comunicación (TICs), se han emanado a gran velocidad, ganando cada vez más espacios y usuarios en las diferentes latitudes y sectores sociales, por consiguiente, se ha dado lugar a la denominada: "Nueva era del conocimiento".

Lo anterior ha traído múltiples consecuencias en el orden social, científico, investigativo, educativo, deportivo, empresarial e incluso tecnológico y como consecuencia se ha originado su diversificación y aplicabilidad en las diferente esferas y sectores sociales, tal es así que la Organización para la Cooperación y el Desarrollo Económico (OCDE), (2020), la define como un proceso social, enfatizando en su importancia y en la necesidad de aprovechar al máximo sus bondades y beneficios sobre todo en los procesos de formativos y de aprendizaje, no solo en el contexto latinoamericano, sino también a nivel mundial.

En este orden estudios realizados por Castells, (2001), Díaz, Pérez, \& Florido, (2011),Valderrama, \& Neme, (2011). Luque \& Galora, (2020), así lo confirman avalando su extrema importancia.

En correspondencia con ello Hernández en el (2003), alerta sobre la importancia de lo que estaba ocurriendo en cuanto al impacto social de la introducción de las TICs, señalando que las TICs, dieron lugar a la "división del mundo en dos humanidades, una que se mueve hacia la sociedad ciberespacial, y otra que vegeta en una economía de subsistencia". Este concepto reviste gran importancia; de acuerdo a ello la sociedad a nivel mundial queda marcada por el desarrollo e introducción de las TICs, pudiendo llegarse a asumir que existe un antes y un después de las TICs, es decir, un entramado social que tiene lugar antes de las TICs y uno posterior a su introducción y aplicación.

Lo cierto es que la introducción de las, ha provocado una revolución en la sociedad moderna, impactando de manera transcendental en las formas de producir el conocimiento y en las propias relaciones y modos de comunicación que se establecen en las diferentes sociedades y comunidades.

\section{Las Tecnologías de la Información y la Comunicación, un acercamiento a la conceptualización y reflexión.}

Ahora bien, ¿Qué entendemos por TICs? Según Castañeda, (2006) en su intervención en la Conferencia impartida en el 3er Congreso ONLINE del Observatorio para la Ciber-Sociedad las define como: "el resultado de las posibilidades creadas por la humanidad en torno a la digitalización de datos, productos, servicios y procesos y de su transportación a través de diferentes medios, a grandes distancias y en pequeños intervalos de tiempo, de forma confiable, y con relación costo-beneficio nunca antes alcanzadas por el hombre". 
Tello, (2007), indica que las TICs, encierra un término que está relacionado con toda forma de comunicación que se emplea en la educación.

Para Díaz, Pérez, \& Florido, (2011), las tecnologías de la Información y Comunicación son: "el conjunto de procesos y productos derivados de las nuevas herramientas (hardware y software), soportes de la información y canales de comunicación relacionados con el almacenamiento, procesamiento y transmisión digitalizada de la información."

Aportes más recientes señalan que aunque aún no existe un concepto claro sobre este término se pueden definir como: "un grupo diverso de prácticas, conocimientos y herramientas, vinculados con el consumo y la transmisión de la información y desarrollados a partir del cambio tecnológico vertiginoso que ha experimentado la humanidad en las últimas décadas, sobre todo a raíz de la aparición de Internet."

En síntesis, esta fuente explica que estamos en presencia de un conglomerado de experiencias digitales que ha avanzado vertiginosamente en la sociedad para producir conocimiento y provocar nuevas formas de interrelación basada entre otros aspectos en el comercio electrónico, la mensajería instantánea, comunidades virtuales, correo electrónico entre otras.

Cruz, M.A., et al (2019), explica en su artículo que coincide con Marqués, (2013), al señalar que: " las TICs se desarrollan a partir de los avances científicos producidos en el ámbito de la informática y de las telecomunicaciones". Destaca que estas resultan de gran utilidad en la búsqueda de información y la gestión Apunta que es : "el conjunto de herramientas, soportes y canales para el proceso y acceso a la información, que forman nuevos modelos de expresión, nuevas formas de acceso y recreación cultural".

Reflexionando en torno a los diferentes conceptos abordados llegamos a establecer que existen elementos coincidentes y comunes en las definiciones ofrecidas; justo estas se relacionan con esta forma novedosa de crear y producir el conocimiento, e incluso las relaciones y modos de comunicación que se da entre los seres humanos en la actualidad, la cual acorta distancias y formas de interrelacionarse. A nuestro modo se trata de una herramienta altamente efectiva, necesaria, de gran aplicabilidad que apunta cada vez más al desarrollo científico, tecnológico y social.

En todo ello no podemos olvidar que la llegada de las TICs, ha provocado el desarrollo social, estamos ante una sociedad cada vez más competitiva, creativa y por ende capaz de generar soluciones encaminadas a dar atención y respuesta a las propias necesidades sociales, en función de lograr el máximo desarrollo posible. Lo importante aquí según criterio de los autores radica precisamente en propiciar el desarrollo de procesos formativos que permitan utilizar la creación y transferencia del conocimiento en alternativas válidas para encontrar y ascender a mejores condiciones de vida y mayor calidad y beneficios tanto a nivel personal como a resultados productivos $\mathrm{y}$ empresariales se refiere. 
Lo paradójico de esto radica en que si bien por un lado resulta muy convincente los excelentes impactos que ha generado y continúan generando las TICs en la sociedad en general, sin embargo no siempre su accesibilidad a pesar de sus altas bondades y positivos efectos se logra de igual forma, la misma aun no resulta equitativa, entre otros factores por las propias brechas que existen en su uso, dadas por el nivel de preparación, competencias y conocimientos para usarlas, por dificultades económicas e incluso geográficas y porque no también, aquellas brechas que se originan por condiciones políticas o gubernamentales.

Lo señalado queda reafirmado por la Organización para la Cooperación y el Desarrollo Económico, (2020), en el informe presentado se destaca que la brecha se produce por el no acceso a computadoras e internet que en muchas ocasiones esta presente en los diferentes sectores sociales a nivel mundial, con énfasis en los sectores más pobres y grupos sociales vulnerables donde la tecnología aun no ha llegado. Todo ello como es ilógico pensar trae serias consecuencias para el sector el desarrollo científico, investigativo, empresarial y educativo.

Es por ello que, en el caso de la Gestión del Talento Humano, continúa siendo un tema de interés la utilización y aplicabilidad de las Tecnologías de la Información y la Comunicación en la generación de alternativas conducentes a optimizar la Gestión del Talento Humano, unido a esto se presenta la necesidad de continuar trabajando por la eliminación de las posibles brechas digitales que pueden existir y que muchas veces ocasionan impiden determinar con claridad qué hacer, ¿cómo hacer y para qué hacer? Atendiendo a ello el Objetivo del presente trabajo se enmarca en establecer la relación que se da entre las Tecnologías de la Información y la Comunicación y la calidad del proceso de Gestión del Talento Humano como base esencial para optimizar los procesos inherentes a esta área.

\section{Importancia y relación de las Tecnologías de la Información y la Comunicación en la Gestión del Talento Humano.}

La formación de un Talento Humano competente, creativo y apto para participar en incluirse progresivamente en el desarrollo social y empresarial sigue constituyendo un foco de muchísimo interés científico y educativo. En correspondencia a lo señalado los procesos formativos de las diferentes carreras buscan encontrar alternativas que mejoren cada vez más la formación y preparación de los futuros egresados.

Una de las herramientas que hace que la Gestión del Talento Humano de una empresa sea realmente competente, radica precisamente en el nivel de preparación y experticia que posean sus empleados para el manejo de la Tecnología de la Información y la Comunicación. Cabe preguntarnos entonces ¿Qué sucede si esto no se alcanza en el proceso formativo? ¿Cómo lograr formar y aportar a la sociedad un profesional competente y apto para el manejo de las TICs, que sepa desenvolverse en el mundo empresarial y que posea las competencias necesarias para provocar impactos positivos en su entidad laboral aportando a la mejora y consecución de resultados productivos positivos? 
Se comparte con Cruz, M.A., et al (2019), al resaltar que actualmente, tener competencias para poder acceder a las Tecnologías de la Información y Comunicaciones (TICs), es un requisito imprescindible, tanto a nivel personal, empresarial $\mathrm{u}$ organizacional. Lo anterior esta dado porque vivimos en una sociedad altamente tecnologizada y aunque no es siempre accesible para todos, cada día se presenta con mayor empuje el uso de la TICs.

En el contexto empresarial manejar adecuadamente las TICs, constituye un elemento dinamizador fundamental de la Gestión de los Talentos Humanos. Su empleo favorece la obtención de mejores resultados, apuntándose a la optimización y desarrollo individual y colectivo y por tanto a la mejora de la gestión en general, de ahí la importancia y relación que se da entre las TICs y la Gestión del Talento Humano.

Al respecto, Roig, (2016), recalca en la necesidad de mejorar las competencias digitales de los trabajadores, para ello propone el desarrollo de un sistema de claves basadas en la tecnología, innovación e investigación. Explica que estas deben darse desde los propios procesos formativos y de enseñanza-aprendizaje, resaltando la importancia de visualizar en ello la aplicación de la tecnología, la innovación y la investigación.

De acuerdo a los estudios de Young, et al (2018), seguidos por Bravo, Chalezquer \& Puche (2018), y Sagrera, Páez \& Polo, (2020), el manejo de las tecnologías se ubica dentro de las competencias necesarias para incidir en la calidad de la Gestión del Talento Humano. Se comparte con los autores precedentes que al poseer estas competencias digitales el profesional estará en condiciones de acceder a la información, al mismo tiempo podrá tener una cultura digital que le ayudará a motivar cambios en la gestión laboral, será más ético y responsable en el manejo de la información; todo lo cual se revertirá positivamente en los resultados de su gestión.

Por tanto, aquellas empresas que logren implementar un sistema de gestión basado en el uso de las TICs y que cuenten además con un talento humano capacitado para su empleo serán por consiguiente instituciones más competitivas, privilegiadas y aptas para establecer la mejora y calidad de sus resultados. En este sentido se reafirma la estrecha relación que se sa entre el uso de las TICs y la calidad de la Gestión de los Talentos Humanos.

\section{Metodología}

En el estudio se utilizó una metodología descriptiva, no experimental. Basada en métodos teóricos dentro de estos el histórico-lógico, analítico -sintético, inductivodeductivo y la revisión documental, todo ello permitió revisar la literatura precedente y establecer los principales argumentos relacionados con el tema. Los criterios de inclusión establecidos tales como: rango de tiempo, resultados y aportes relacionados con el tema de la Gestión de los Talentos Humanos y el Uso de las TICs, entre otros favorecieron la calidad del trabajo realizado. 


\section{Resultados y Discusión}

El análisis de los referentes teóricos consultados nos permite establecer cuatro resultados claves:

1. La revisión sistemática de la literatura permite refirmar que en el contexto actual el uso de las Tecnologías de la Información y la Comunicación constituye una herramienta fundamental e imprescindible para perfeccionar la Gestión del Talento Humano en las diferentes instituciones u empresas. Lo cual se corresponde con los aportes de Roig, (2016), Young, et al (2018), seguidos por Bravo, Chalezquer \& Puche (2018), Cruz, M.A., et al (2019) y Sagrera, Páez \& Polo, (2020),

2. Coincidiéndose con Hernández, (2003). Cortés, (2015), se establece que para el logro de lo anterior se requiere trabajar en la eliminación de las brechas digitales, que aún persisten; ello implica lograr la accesibilidad a computadoras, internet y a la tecnología en sí, eliminando la desigualdad existente producto del propio desarrollo económico y social que no siempre permite la asequibilidad y accesibilidad para todos.

3. Se hace necesario además restructurar los procesos formativos considerando no solo en ello el desarrollo de estrategias de orientación y capacitación, sino además la puesta en marcha de planes y programas que, unido a la mejora de las mallas curriculares en las diferentes carreras, favorezcan la accesibilidad permitiendo acortar las brechas en los procesos de enseñanza-aprendizaje y en los medios tecnológicos para poder acceder al uso de la tecnología.

4. Se requiere intensificar el desarrollo de procesos de diagnóstico empresarial, que develen las competencias digitales de los talentos humanos que componen la empresa y de esta forma desarrollar un sistema de capacitación que se atempere a las necesidades de los trabajadores.

1. Derivado del análisis teórico realizado se procede a presentar una propuesta de estrategia basada en cuatro aspectos fundamentales: Diagnóstico, Planificación, Aplicación y Retroalimentación, la cual favorecerá detectar tempranamente donde se encuentran las limitaciones de los Talentos Humanos y planificar estrategias de decapitación en función de mejorar sus competencias para el manejo adecuado de las TICs, 


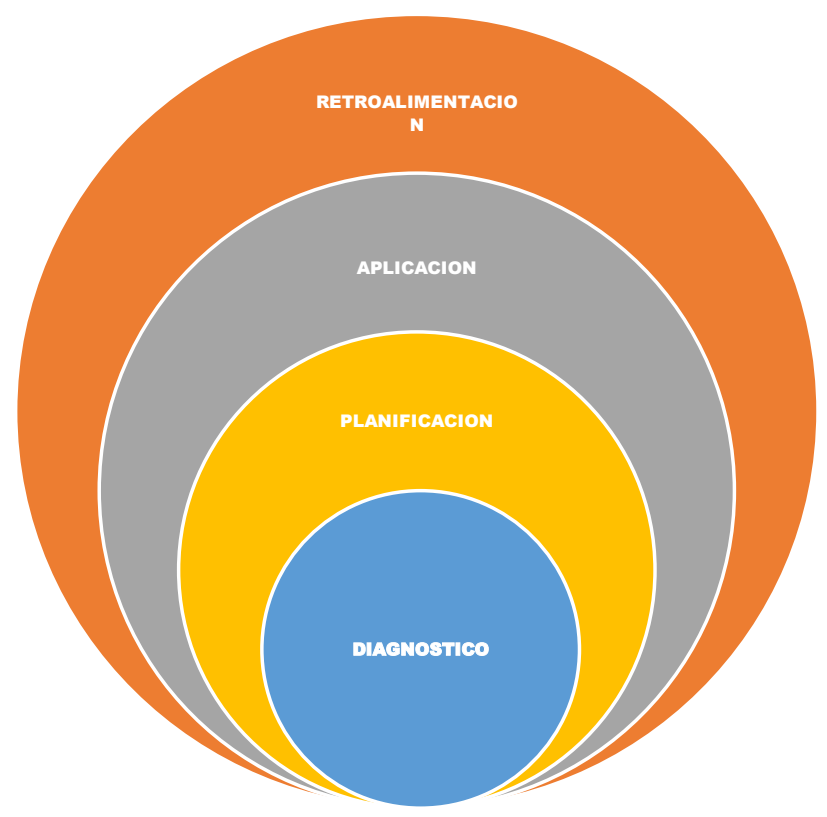

Elaborado por: Autores (2022).

\section{Conclusiones}

- La revisión sistemática de la literatura permitió confirmar la importancia de utilizar la Tecnología de la Información y la Comunicación como herramienta fundamental para la mejora de la Gestión del Talento Humano en las diferentes instituciones u empresas.

- Se aporta una estrategia de desarrollo posible a aplicar en las diferentes para la optimización del desarrollo de las competencias y manejo de la las TICs, con vista al perfeccionamiento de la Gestión del Talento Humano

\section{Referencias Bibliográficas}

Bravo, M. C. M., Chalezquer, C. S., \& Puche, J. S. (2018). Desarrollo de competencias digitales en comunidades virtuales: un análisis de "ScolarTIC". Prisma Social: revista de investigación social, (20), 129-159.

Castañeda, AE., (2006) Las tecnologías de la información y las comunicaciones (TICs) en el proceso de enseñanza aprendizaje a comienzos del siglo XXI:¿Problema didáctico o epistemológico? Conference: 3er Congreso ONLINE del Observatorio para la Ciber-Sociedad DOI:10.13140/RG.2.1.4003.0884

Castells, M. (2001). La Galaxia Internet. Ed. Areté. Barcelona, España. ISBN: 84-01$34157-4$

Cortés, A. X., (2015). El impacto de la tecnología en el ámbito social y en la desigualdad (The Impact of Technology in the Social Sphere and 
Inequality). $I y D, 2(2)$, $16-20$. https://doi.org/10.26620/uniminuto.inclusion.2.2.2015.16-20

Cruz, M.A., Pozo, M.A., Aushay, H.R. y Arias, A.D. (2019). Las Tecnologías de la Información y de la Comunicación (TIC) como forma investigativa interdisciplinaria con un enfoque intercultural para el proceso de formación estudiantil. e-Ciencias de la Información, 9(1). doi: https://doi.org/10.15517/eci.v1i1.33052

Díaz, J., Pérez, A., \& Florido, R., (2011). Impacto de las Tecnologías de la Información y las Comunicaciones (TIC) para disminuir la brecha digital en la sociedad actual. Cultivos Tropicales, 32(1), 81-90. Recuperado en 07 de enero de 2022, de http://scielo.sld.cu/scielo.php?script=sci_arttext\&pid=S025859362011000100009\&lng=es\&tlng=es.

Hernández, A., (2003). Modernidad y tecnología o de la brecha entre cultura y tecnología en las sociedades modernas. Lecturas en Humanidades: Sociedad y Tecnología I (10)15-32.

Luque, A., Galora, R. P.(2020) Impacto de la tecnología en la sociedad: el caso de Ecuador. Revista Ciencias Pedagógicas e Innovación Volumen VII, No 2 (enerojunio 2020), pp. 40-47 http://dx.doi.org/10.26423/rcpi.v7i2.299

Marqués, P. (2013). Impacto de las TIC en Educación: Funciones y limitaciones. Rev. 3C TIC, 1(3). Recuperado de https://www.3ciencias. com/wpcontent/uploads/2013/01/impacto-de-las-tic.pdf

Roig, R., (ed.). (2016) Tecnología, innovación e investigación en los procesos de enseñanza-aprendizaje. Barcelona: Octaedro, 2016. ISBN 978-84-9921-848-9, 3173 p.

OCDE (2020), Making the Most of Technology for Learning and Training in Latin America, https://doi.org/10.1787/ce2b1a62-en. (C) 2020 OCDE, París

Segrera, JR., Paez-, H D., Polo, Aa., (2020), Competencias digitales de los futuros profesionales en tiempos de pandemia Utopía y Praxis Latinoamericana, vol. 25, núm. Esp.11, 2020 Universidad del Zulia, Venezuela Disponible en: https://www.redalyc.org/articulo.oa?id=27964922015 DOI: https://doi.org/10.5281/zenodo.4278352

Tello, E (2007). «Las tecnologías de la información y comunicaciones (TIC) y la brecha digital: Su impacto en la sociedad de México» [artículo en línea]. Revista de Universidad y Sociedad del Conocimiento (RUSC). Vol. 4, n. ${ }^{\circ}$ 2. UOC. [Fecha de consulta: 7/1/22]. ISSN 1698-580X

Valderrama, AL., \& Neme, O., (2011). Efecto de las tecnologías de la información y comunicaciones (TIC) en las exportaciones manufactureras en 
México. Economía UNAM, 8(24), 99-122. Recuperado en 07 de enero de 2022, de http://www.scielo.org.mx/scielo.php?script=sci_arttext\&pid=S1665952X2011000300005\&lng=es\&tlng=es

Young, T., Jimenez, F., Segrera, J., Agenor, G., Albor, V. Y Zambrano, M., (2018). "Innovación Tecnológica en la empresa de Manufactura Cannon de Colombia S.A. en Barranquilla - Colombia", en: Gestión del Conocimiento, Una perspectiva Multidisciplinaria Volumen 8. Libro resultado de investigación, bajo la coordinación del Dr. Víctor Meriño y el aval del Fondo Editorial Universitario Servando Garcés. Coro, Venezuela. p.p. 201- 215. https://concepto.de/tics/. Concepto de TICs. Consultado 20/1/22.

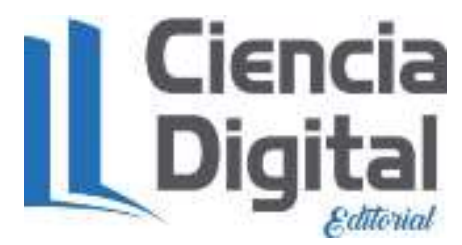




\section{PARA CITAR EL ARTÍCULO INDEXADO.}

Gavilanes Sagñay, M. A., Gavilanes-Sagnay, F., \& Chávez Granados, N. A. (2022). Las tecnologías de la información y la comunicación y su relación con la gestión del talento humano. ConcienciaDigital, 4(4.2), 101-111. https://doi.org/10.33262/concienciadigital.v4i4.2.1961

\section{LCiencia}

El artículo que se publica es de exclusiva responsabilidad de los autores y no necesariamente reflejan el pensamiento de la Revista Conciencia Digital.

El artículo queda en propiedad de la revista y, por tanto, su publicación parcial y/o total en otro medio tiene que ser autorizado por el director de la Revista Conciencia Digital.

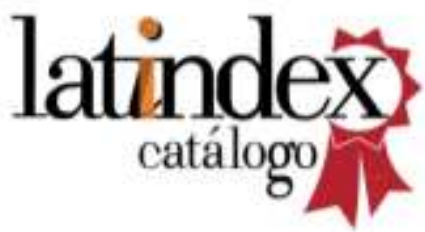

